ARTICLE

\title{
Kinetic Analysis of Weakly Coupled Systems Using Probability Density Function of Coupling Coefficient Obtained by Monte Carlo Method
}

\author{
Toru OBARA* and Hiroki TAKEZAWA \\ Research Laboratory for Nuclear Reactors, Tokyo Institute of Technology, \\ 2-12-1-N1-19 Ookayama, Meguro-ku, Tokyo 152-8550, Japan
}

\begin{abstract}
A kinetic analysis code for weakly coupled systems is developed. It is based on the theory of a time-dependent integral neutron transport equation. Using this method, kinetic analyses in the super prompt critical condition are performed for a nuclear pumped laser experiment reactor, which is a weakly coupled system of fast-pulse cores and a thermal laser module. The results show that there is not only a delay in the pulse peak in the thermal laser module in response to the fast-pulse cores but also a similar delay in the pulse peak in outer region of the thermal laser module in response to the inner region of the thermal laser module. Our analysis is demonstrated that this method is effective for space-dependent kinetic analysis in weakly coupled systems.
\end{abstract}

KEYWORDS: kinetic analysis, weakly coupled systems, probability density, coupling coefficient, Monte Carlo

\section{Introduction}

One-point kinetic theory is widely used in kinetic analysis. The method is feasible for the analysis of most conventional reactors. In some cases, however, it is necessary to perform space-dependent kinetic analysis for weakly coupled systems. In such cases, the point kinetic method is not feasible. Examples of weakly coupled systems in which kinetic analysis is needed are super prompt critical kinetic analysis with several fuel solution tanks and kinetic analysis of experiment reactors for nuclear-pumped lasers, which have coupled, fast-pulse cores and a thermal laser module. No effective and practical method to do kinetic analysis for such system has been developed. For the analysis of such systems, a space-dependent kinetic method for weakly coupled systems is needed.

For the space-dependent kinetic analysis in the super prompt condition, a kinetic analysis code has been developed and validiated. ${ }^{1,23}$ This code is based on the time-dependent integral neutron transport equation using the time-dependent probability density function of the coupling coefficient. ${ }^{4)}$ Some kinetic analyses have been performed by the developers for weakly coupled fast-pulse cores and thermal laser modules by using the code. The space-dependent kinetic analyses were performed by dividing the reactor into three regions, two corresponding to the pulse core and one to the thermal laser module. In this case, the reactor power increased rapidly. In response to a negative feedback effect of the cores, the power of each region reached a peak value and then decreased. The analyses showed that the reactor period after reactivity insertion depended on the neutronic characteristics of the thermal laser module, that the time of the power peak in each region dif-

*Corresponding author, E-mail:tobara@nr.titech.ac.jp fered, and that the time of the power peak in the thermal laser module region was delayed from that of the fast-pulse cores. In the experiment reactor, the change in the power density distribution in the thermal laser module during the transient is expected to have some effect on laser oscillation in the module. Therefore, to determine the space distribution of fission power during transient in the reactor is quite important. It can be expected that in the thermal laser module, the power density distribution during the transient can be changed because of the reactor design, in which the fast-pulse cores are set inside the thermal laser module.

The purpose of this study was to demonstrate that the developed method is effective for space-dependent kinetic analysis of weakly coupled systems in the super prompt critical condition. We attempted to show that the change in the power density distribution during the super prompt critical condition in the thermal laser module can be analyzed in the experiment reactor of a nuclear-pumped laser, which is a coupled system of fast-pulse cores and a thermal laser module.

\section{Methodology}

The developed code is based on the theory of the time-dependent integral transport equation and the probability density function of the coupling coefficient. ${ }^{2,4)}$

A weakly coupled system was divided into multiple regions to analyze changes in the fission power of each region at the supercritical condition. The analyses of the first pulse focused on the current study, so only prompt neutrons are considered in the code.

The time-dependent integral transport equation in this case can be expressed by the following equation by using the probability density function of the coupling coefficient between the regions. 


$$
N_{i}(t) \cong \sum_{j=1}^{n} \int_{t-t_{c}^{p}}^{t} \alpha_{i j}^{p}\left(t-t^{\prime}\right) N_{j}\left(t^{\prime}\right) d t^{\prime},
$$

where $N_{i}(t)$ is the number of fissions in unit time in region $i$ at time $t, \alpha_{i j}^{p}(t)$ is the probability density that a fission occurs in region $i$ time $t$ after a fission occurs in region $j$ by prompt neutrons, $t_{c}^{p}$ is the time when stepwise positive reactivity is inserted, and $n$ is the total number of regions. The probability density can be said to be a probability density function of coupling coefficient. The probability in which a fission occurs in region $i$ until time $\tau$ after a fission occurs in region $j$ by prompt neutrons is expressed in the following equation,

$$
C_{i j}^{p}(\tau) \equiv \int_{0}^{\tau} \alpha_{i j}^{p}\left(\tau^{\prime}\right) d \tau^{\prime}
$$

If $\alpha_{i j}^{p}(t)$ or $C_{i j}^{p}(\tau)$ are known, it is possible to calculate the number of fissions per unit time in each region after the reactivity insertion by using the finite difference method.

$C_{i j}^{p}(\tau)$ can be obtained by neutron transport Monte Carlo calculations. By taking a tally of the time between the fission events in non-analog Monte Carlo calculations, the average value of probability in which a fission occurs in region $i$ until time $\tau$ after a fission occurs in region $j$ by prompt neutrons is obtained by the following equation.

$$
\overline{C_{i j}^{p}(\tau)}=\frac{1}{N_{\text {tally }}} \sum_{L=1}^{N_{\text {tally }}}\left[\frac{\sum_{0 \leq \tau \prime \leq \tau}\left(\frac{\sigma_{f x}\left(E_{i n}\right)}{\sigma_{t x}\left(E_{\text {in }}\right)}\right) w_{i j}^{i n}}{\sum_{L} \frac{W_{0 j x \prime}}{v_{p x \prime}\left(E_{m}\right)}}\right],
$$

where $L$ shows the index number of tally batches in the eigenvalue Monte Carlo calculation, i.e. the index which shows each random walk of neutron, $N_{\text {tally }}$ is the total number of tally batches, $\sigma_{f x}\left(E_{i n}\right)$ and $\sigma_{t x}\left(E_{i n}\right)$ are the microscopic fission cross section and the microscopic total cross section of nuclide $x$ at energy $E_{i n}$ respectively, $w_{i j}^{i n}$ is the weight of the neutron before collision whose initial point is in region $j$ and causes a collision in region $i, W_{0 j x}$ is the weight of a fission source neutron in region $\mathrm{j}$ from nuclide $x$, and $v_{p x}\left(E_{m}\right)$ is the number of prompt fission source neutrons produced by the fission of nuclide $x^{\prime}$ by the incoming mother neutrons with energy $E_{m} . \Sigma_{0 \leq \tau \leq \tau}$ in the numerator means the summation of the values in the collisions at time $\tau^{\prime}$ which satisfies $0 \leq \tau^{\prime} \leq \tau$. Continuous Energy Monte Carlo code MVP2.0 $0^{5)}$ was modified to calculate $\overline{C_{i j}^{p}(\tau)}$ in the eigenvalue calculation using nuclear data library JENDL-3.3. ${ }^{6}$

From Eq. (1), the difference equation when the reactor is critical at time $t<0$ and becomes super critical at $t=0$ is obtained as the following.

$$
\begin{aligned}
N_{i}(k \Delta t) \cong & \left\{\int_{-\infty}^{0} \widetilde{\alpha_{l j}^{p}}\left(k \Delta t-t^{\prime}\right) N_{j 0} d t^{\prime}\right. \\
& \left.\quad+\int_{0}^{k \Delta t} \alpha_{i j}^{p}\left(k \Delta t-t^{\prime}, \mathbf{T}\left(t^{\prime}\right)\right) N_{j}\left(t^{\prime}\right) d t^{\prime}\right\} \\
= & \sum_{j=1}^{n}\left\{N_{j 0}\left[\widetilde{C_{l j}^{p}}\left(\tau^{\prime}\right)\right]_{k \Delta t}^{\infty}\right. \\
& \left.+\sum_{k^{\prime}=0}^{k-1} N_{j}\left(k^{\prime} \Delta t\right)\left[C_{i j}^{p}\left(\tau^{\prime}, \mathbf{T}_{k^{\prime}}\right)\right]_{\left(k-k^{\prime}-1\right) \Delta t}^{\left(k-k^{\prime}\right) \Delta t}\right\}
\end{aligned}
$$

where $\widetilde{\alpha_{l j}^{p}}(\tau)$ and $\widetilde{C_{l j}^{p}}(\tau)$ are $\alpha_{i j}^{p}(\tau)$ and $C_{i j}^{p}(\tau)$ respectively, at the critical steady state condition, in which the temperature is initial uniform temperature, $N_{j 0}$ is the number of fissions in unit time in region $\mathrm{j}$ in the critical condition, $\mathbf{T}\left(t^{\prime}\right)$ is the vector whose element shows temperature of each region in the system at time $t^{\prime}, \Delta t$ is the width of the time step, $k$ is the number of time steps, and $\mathbf{T}_{k}$, is the vector whose element shows temperature of each region in the system at time step $k^{\prime}$. The $\alpha_{i j}^{p}(\tau)$ and $C_{i j}^{p}(\tau)$ depend on the temperature vector. For the practical calculation, the change of temperature of a region which has little effect on reactivity can be neglected to simply the analysis. The code to calculate the number of fissions per unit time in each region at each time step by using the Runge-Kutta method has been developed. ${ }^{3)}$

The reactivity feedback effect during the transient can be treated to calculate $C_{i j}^{p}\left(\tau^{\prime}, \mathbf{T}\right)$ in different temperature distributions. In the calculation, it is assumed that heat transfer between the different regions is negligible because the transient period is very short. A table of values of $C_{i j}^{p}\left(\tau^{\prime}, \mathbf{T}\right)$ for different fast-pulse core temperatures has been prepared. The temperature rise for each region is calculated by the integral power and its heat capacity. At each time step, interpolated values obtained from the table at the appropriate temperature are used for the transient calculation. By the code, the change of fission power in each region is calculated after stepwise reactivity insertion from the critical condition.

\section{Kinetic Analysis of a Nuclear Pumped Laser Experiment Reactor as an Example of a Weak- ly Coupled System}

One example of a weakly coupled system is the experiment reactor for a nuclear pumped laser that was developed in IPPE, Russia. ${ }^{7)}$ The reactor consists of two parts. One is two fast-pulse cores made of highly enriched uranium metal, and the other is a thermal laser module, which surrounds the fast-pulse cores. The neutron spectrum in the fast-pulse cores is so hard. In the thermal laser module, a polyethylene moderator is used and the amount of uranium metal is small, so the neutron spectrum in the thermal laser module is so soft. There are void regions between the two regions, so the coupling of the two regions is weak.

The developed code is effective for the transient analysis when the reactor becomes prompt super critical from the critical condition by stepwise reactivity insertion. Some 


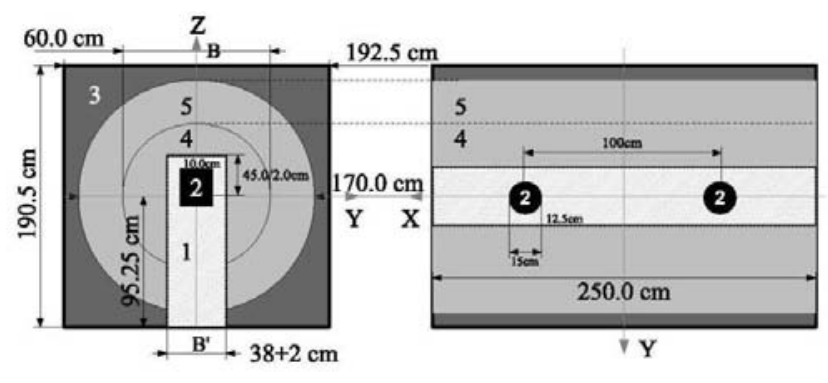

Fig. 1 Calculation geometory of Nuclear Pumped Laser Experiment Reactor (1: Void, 2: Fast pluse cores, 3: Polyethylene reflector, 4: Inner region of thermal laser module, 5: Outer region of thermal laser module)

analysis has been performed by using the code. ${ }^{3)}$ In the analysis, the reactor has been divided into three regions, one fast-pulse core, another fast-pulse core, and the thermal laser module. It was shown by the analysis that there was some difference in the power change during the transient between the fast-pulse cores and the thermal laser module.

The volume of the thermal laser module is large, so it is expected that there would also be a difference in the power change for the thermal laser module. In the experiment for laser oscillation, it is expected that such a difference or delay in reaching the maximum power in each region in the thermal laser module can have a remarkable effect. In the study, analysis was performed by dividing the thermal laser module into two regions to determine whether such a delay could be analyzed by the method.

The geometry of the reactor used for the analysis is shown in Fig. 1. The reactor consisted of two parts. First, there were two fast-pulse cores made of highly enriched uranium. In the analysis, the uranium enrichment was set to $100 \%$. The other part was a thermal laser module consisting of laser cell tubes and a polyethylene moderator. The laser cell tubes were made of stainless steel tubes with an approximate tube diameter of $5 \mathrm{~cm}$. The inside surfaces of the tubes were coated with highly enriched uranium metal. A polyethylene moderator was set between the tubes. The laser module was a subcritical thermal core. The design parameters of the fast-pulse cores and the laser module are shown in Table $\mathbf{1 .}$

Kinetic analysis was performed for the reactor. In the analysis, an eigenvalue calculation was performed to obtain the effective multiplication factor $k_{\text {eff }}$. The initial condition was set by changing the number of neutrons per fission $v$ to $v / k_{\text {eff }}$. At time $t=0$, positive reactivity was inserted by changing the value in a stepwise manner to $v$, and kinetic calculations were performed after reactivity insertion. The reactor power at the initial condition was set to $1 \mathrm{~W}$.

The reactor was divided into three regions in the analysis. The first region was two fast-pulse cores. The thermal core for the laser module was divided into two regions in a radial direction. The second region was an inner part of the thermal core, and the third region was the outer part of the thermal core (Fig. 1). The change in fission power was calculated for each region. The initial temperature was set to $300 \mathrm{~K}$. It was assumed that there was no heat transfer between the regions during the transient. The $\overline{C_{i j}^{p}(\tau)}$ values were calculated for
Table 1 Design Parameters of Experimental Reactor for Nuclear Pumped Laser

\begin{tabular}{ll}
\hline Fast pulse cores & \\
Material & Uranium metal \\
Enrichment & $100 \%$ \\
Core height & $15 \mathrm{~cm}$ \\
Core radius & $7.5 \mathrm{~cm}$ \\
Number of cores & 2 \\
& \\
Laser cell module & $\mathrm{SS316}$ \\
Material of laser cell tube & $49 \mathrm{~mm} / 50 \mathrm{~mm}$ \\
Inner / Outer diameter of the laser cell & Uranium metal \\
Inner coating of laser cell & $100 \%$ \\
Enrichment of uranium & $5 \mu \mathrm{m}$ \\
Thickness of the coating & Ar:Xe=200:1 \\
Laser gas medium & $1 \mathrm{~atm}$ \\
Laser gas pressure & $55 \mathrm{~mm}$ \\
Laser cell pitch & Polyethylene \\
Moderator between the laser cell tubes & \\
\hline
\end{tabular}

temperatures of $300 \mathrm{~K}$ and $800 \mathrm{~K}$. The MVP 2.0 and JENDL-3.3 nuclear data library were used to calculate the initial condition. The $\overline{C_{i j}^{p}(\tau)}$ values were obtained by the modified MVP2.0 with the JENDL-3.3 nuclear data library. The kinetic calculation was performed by using the kinetic code developed using the $\overline{C_{i j}^{p}(\tau)}$ values.

\section{Calculation Results and Discussion}

The effective multiplication factor of the initial condition was found to be 1.1057. Calculated changes in fission power after the reactivity insertion at each region are shown in Fig. 2. The calculation results show that there are some delays in the power peak between the regions. Table 2 shows the delay in the power peak for each region in the thermal laser module from the power peak of the fast cores. The table also shows the full width of half maximum of the peaks. The results show that the power peak of the thermal laser module delays from $4.0 \times 10^{-3} \mathrm{~s}$ to $4.1 \times 10^{-3} \mathrm{~s}$ from the power peak of fast-pulse cores. The results also show that the power peak of the outer region of the thermal laser module is delayed by approximately $0.1 \times 10^{-3} \mathrm{~s}$ from the power peak of the inner region of the thermal laser module. This finding indicates that the time of the power peak depends on the position in the thermal laser module.

As shown previously, ${ }^{3)}$ the time constant of the power increase of fast cores after the reactivity insertion depends on the neutronic characteristics of the thermal laser module. Because of differences in the neutron generation time between the fast-pulse cores and the thermal laser module, transient behavior after the reactor becomes supercritical is different. The fission neutrons in the thermal laser module and fast neutrons from fast-pulse cores cause fissions after the neutron-slowing process in the thermal laser module. This process causes a delay in power peaking in the thermal laser module. In this study, it was shown that the time of the power peak in the thermal laser module differs from that in the different region. This result suggests that there is a wave of the fission power peak that moves from inner to outer 


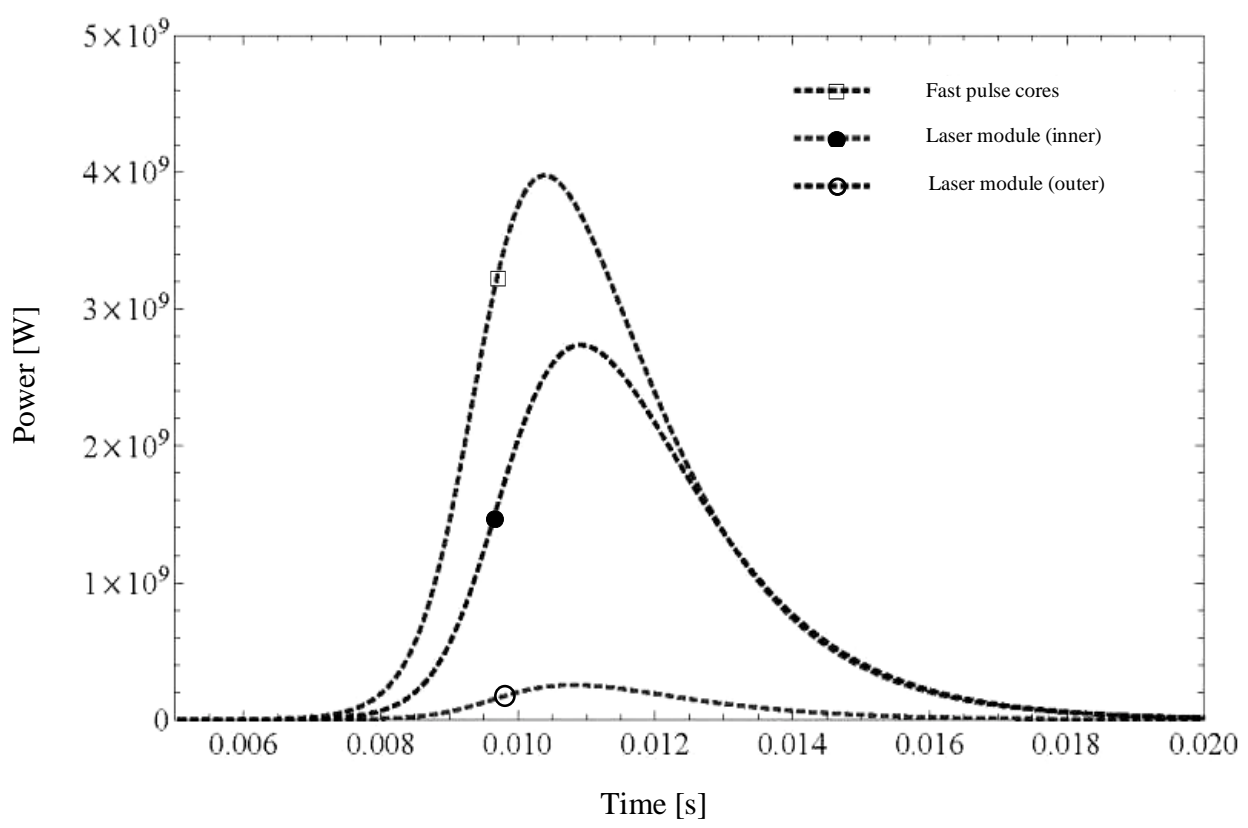

Fig. 2 Change of power after reactivity insertion

Table 2 Fission power peaks after reactivity insertion

\begin{tabular}{cccc}
\hline & $\begin{array}{c}\text { Fast pulse } \\
\text { cores }\end{array}$ & $\begin{array}{c}\text { Inner laser } \\
\text { module }\end{array}$ & $\begin{array}{c}\text { Outer laser } \\
\text { module }\end{array}$ \\
\hline $\begin{array}{c}\text { Time of peak after } \\
\text { the peak of } \\
\text { fast-pulse cores } \\
{[\mathrm{s}]}\end{array}$ & - & $4.0 \times 10^{-3}$ & $4.1 \times 10^{-3}$ \\
\hline $\begin{array}{c}\text { Full width at half } \\
\text { maximum [s] }\end{array}$ & $3.4 \times 10^{-3}$ & $3.6 \times 10^{-3}$ & $3.6 \times 10^{-3}$ \\
\hline
\end{tabular}

areas of the thermal laser module. It is expected that the space and time dependency of fission power in the thermal laser module may affect the laser oscillation. It is meaningful that it is possible to analyze such space and time dependency by using the developed code. In this study, the thermal region was divided into only two regions. It is expected that more detailed space and time-dependent kinetic analysis would be possible by dividing the thermal laser module into more regions. Such detailed analysis may make it possible to observe in detail the movement of the fission power wave peak in the thermal laser module.

\section{Conclusions}

A new kinetic analysis code has been developed using the probability density function of the coupling coefficient based on the time-dependent integral neutron transport equation. The method makes it possible to perform kinetic analysis of weakly coupled systems, for which there was no practical method before. Kinetic analysis was performed for a nuclear pumped laser experiment reactor, which was a weakly coupled system of fast-pulse cores and a thermal laser module. The results showed that after insertion of positive reactivity to make the reactor super critical, there was not only some delay in the power peak of the thermal laser module to the fast-pulse cores, but also a delay in the power peak in the outer region of the thermal laser module to the inner region. These findings indicate that there is space and time dependency of the neutron fission power peak in the thermal laser module during the transient. Based on the present analysis, it appears that the code developed can be useful for kinetic analysis in weakly coupled systems. By dividing the region more, it will be possible to perform more detailed space and time-dependent analysis.

\section{References}

1) H. Takezawa, T. Obara, "Transient Parameters of the Integral Kinetic Model for Weakly Coupled Systems,” Trans. Am. Nucl. Soc., 99, 762 (2008).

2) H. Takezawa, T. Obara, "Calculation of the Integral Kinetic Model Transient Parameters by the Monte Carlo Method for Space-Dependent Kinetic Analysis,” Nucl. Sci. Eng., 164, 80-86 (2010).

3) H. Takezawa, T. Obara, "New Approach to Space-Dependent Kinetic Analysis Based on the Integral Kinetic Model with the Monte Carlo Method," submitted to Nucl. Sci. Eng.

4) O. F. Kukharchuk et al., "Coupled Fast-Thermal Reactor System: Theory and Experiment," Proc. of International Topical Meeting on Advances in Reactor Physics and Mathematics and Computation into the Next Millennium (PHYSOR 2000), Pittsburgh, Pennsylvania, 7-11 May (2000).

5) Y. Nagaya et al., MVP/GMVP II: General Purpose Monte Carlo Codes for Neutron and Photon Transport Calculations Based on Continuous Energy and Multigroup Method, JAERI 1348 (2005).

6) K. Shibata et al., "Japanese Evaluated Nuclear Data Library Version 3 Revision-3: JENDL-3.3,” J. Nucl. Sci. Technol., 39[11],1125-1136 (2002).

7) P. P. D’yanchenko et al., "STAND B Reactor-Laser System," Atom. Energy, 88[5], 352-357 (2002). 